\title{
Integral Transforms of Functions to Be in a Class of Analytic Functions Using Duality Techniques
}

\author{
Satwanti Devi and A. Swaminathan \\ Department of Mathematics, Indian Institute of Technology, Roorkee, Uttarakhand 247 667, India \\ Correspondence should be addressed to A. Swaminathan; mathswami@gmail.com
}

Received 26 February 2014; Accepted 13 May 2014; Published 1 July 2014

Academic Editor: Hari M. Srivastava

Copyright ( 2014 S. Devi and A. Swaminathan. This is an open access article distributed under the Creative Commons Attribution License, which permits unrestricted use, distribution, and reproduction in any medium, provided the original work is properly cited.

Let $W_{\beta}(\alpha, \gamma), \beta<1$, denote the class of all normalized analytic functions $f$ in the unit disc $\mathbb{D}=\{z \in \mathbb{C}:|z|<1\}$ such that $\operatorname{Re}\left(e^{i \phi}\left((1-\alpha+2 \gamma)(f / z)+(\alpha-2 \gamma) f^{\prime}+\gamma z f^{\prime \prime}-\beta\right)\right)>0, z \in \mathbb{D}$, for some $\phi \in \mathbb{R}$ with $\alpha \geq 0, \gamma \geq 0$, and $\beta<1$. Let $M(\xi), 0 \leq \xi \leq 1$, denote the Pascu class of $\xi$-convex functions given by the analytic condition $\operatorname{Re}\left(\left(\xi z\left(z f^{\prime}(z)\right)^{\prime}+(1-\xi) z f^{\prime}(z)\right) /\left(\xi z f^{\prime}(z)+(1-\xi) f(z)\right)\right)>0$ which unifies the classes of starlike and convex functions. The aim of this paper is to find conditions on $\lambda(t)$ so that the integral transform of the form $V_{\lambda}(f)(z)=\int_{0}^{1} \lambda(t)(f(t z) / t) d t$ carry functions from $W_{\beta}(\alpha, \gamma)$ into $M(\xi)$. As for the applications, for specific values of $\lambda(t)$, it is found that several known integral operators carry functions from $W_{\beta}(\alpha, \gamma)$ into $M(\xi)$. The results for a more generalized operator related to $V_{\lambda}(f)(z)$ are also given.

\section{Introduction}

Let $\mathscr{A}$ denote the class of all functions $f$ analytic in the open unit disc $\mathbb{D}=\{z \in \mathbb{C}:|z|<1\}$ with the normalization $f(0)=f^{\prime}(0)-1=0$, and let $\mathcal{S}$ be the class of functions $f \in \mathscr{A}$ that are univalent in $\mathbb{D}$. A function $f \in \mathcal{S}$ is said to be starlike $\left(S^{*}\right)$ or convex $(\mathscr{C})$, if $f$ maps $\mathbb{D}$ conformally onto the domains, respectively, starlike with respect to origin and convex. Note that in $\mathbb{D}$, if $f \in \mathscr{C} \Leftrightarrow z f^{\prime} \in S^{*}$ follows from the well-known Alexander theorem (see [1] for details). A useful generalization of the class $S^{*}$ is the class $S^{*}(\sigma)$ that has the analytic characterization $S^{*}(\sigma)=\left\{f \in A: \operatorname{Re}\left(z f^{\prime} / f\right)>\right.$ $\sigma ; 0 \leq \sigma<1\}$ and $S^{*}(0) \equiv S^{*}$. Various generalizations of classes $S^{*}$ and $\mathscr{C}$ are abundant in the literature. One such generalization is the following.

A function $f \in \mathscr{A}$ is said to be in the Pascu class of $\alpha$ convex functions of order $\sigma$ if [2]

$$
\begin{aligned}
\operatorname{Re} \frac{\alpha z\left(z f^{\prime}(z)\right)^{\prime}+(1-\alpha) z f^{\prime}(z)}{\alpha z f^{\prime}(z)+(1-\alpha) f(z)} & >\sigma, \\
0 \leq \alpha & \leq 1,
\end{aligned}
$$

or in other words

$$
\alpha z f^{\prime}(z)+(1-\alpha) f(z) \in S^{*}(\sigma) .
$$

This class is denoted by $M(\alpha, \sigma)$. Even though this class is known as Pascu class of $\alpha$-convex functions of order $\sigma$, since we use the parameter $\alpha$ for another important class, we denote this class by $M(\xi, \sigma), 0 \leq \xi \leq 1$, and we remark that, in the sequel, we only consider the class $M(\xi):=M(\xi, 0)$. Clearly, $M(0)=S^{*}$ and $M(1)=\mathscr{C}$, which implies that this class $M(\xi)$ is a smooth passage between the classes of starlike and convex functions.

The main objective of this work is to find conditions on the nonnegative real valued integrable function $\lambda(t)$ satisfying $\int_{0}^{1} \lambda(t) d t=1$, such that the operator

$$
F(z)=V_{\lambda}(f)(z):=\int_{0}^{1} \lambda(t) \frac{f(t z)}{t} d t
$$

is in the class $M(\xi)$. Note that this operator was introduced in [3]. To investigate this admissibility property, the class to which the function $f$ belongs is important. Let $W_{\beta}(\alpha, \gamma)$, where $\alpha \geq 0, \gamma \geq 0$, and $\beta<1$, denote the class of all 
normalized analytic functions $f$ in the open unit disc $\mathbb{D}$ such that

$$
\operatorname{Re}\left(e^{i \phi}\left((1-\alpha+2 \gamma) \frac{f}{z}+(\alpha-2 \gamma) f^{\prime}+\gamma z f^{\prime \prime}-\beta\right)\right)>0,
$$$$
z \in \mathbb{D}
$$

for some $\phi \in \mathbb{R}$. This class and its particular cases were considered by many authors so that the corresponding operator given by (3) is univalent and in $M(\xi)$ for some particular values of $\alpha, \beta, \gamma$, and $\xi$. This work was motivated in [3] by studying the conditions under which $V_{\lambda}\left(W_{\beta}(1,0)\right) \subset$ $M(0)$ and was generalized in [4] by studying the case $V_{\lambda}\left(W_{\beta}(\alpha, 0)\right) \subset M(0)$. Similar situation for the convex case, namely, $V_{\lambda}\left(W_{\beta}(1,0)\right) \subset M(1)$, was initiated in [5]. After several generalizations by many authors, recently, the conditions under which $V_{\lambda}\left(W_{\beta}(\alpha, \gamma)\right) \subset M(0)$ were obtained in [6] and the corresponding results for the convex case so that $V_{\lambda}\left(W_{\beta}(\alpha, \gamma)\right) \subset M(1)$ were obtained in [7]. Applications involving several well-known integral transforms were studied in $[6,7]$ (see also [8]). For all of the literatures involving the complete study in this direction so far, we refer to [6-9] and the references therein.

In this work, we find conditions on $\lambda(t)$ so that $V_{\lambda}\left(W_{\beta}(\alpha, \gamma)\right) \subset M(\xi)$ using duality techniques which are presented in Section 2. As applications, in Section 3, we consider particular values for $\lambda(t)$ in (3) so that results for some of the well-known integral operators can be deduced. A more generalized operator introduced in [5] is considered in Section 4 for similar type of results.

First, we underline some preliminaries that are useful for our discussion. We introduce two constants $\mu \geq 0$ and $\nu \geq 0$ satisfying $[6,10]$

$$
\mu+\nu=\alpha-\gamma, \quad \mu \nu=\gamma .
$$

When $\gamma=0$, then $\mu$ is chosen to be 0 , in which case $\nu=$ $\alpha \geq 0$. When $\alpha=1+2 \gamma$, (5) yields $\mu+\nu=1+\gamma=1+\mu \nu$, or $(\mu-1)(1-\nu)=0$, and leads to two cases as follows.

(i) For $\gamma>0$, then, choosing $\mu=1$ gives $\nu=\gamma$.

(ii) For $\gamma=0$, then, $\mu=0$ and $\nu=\alpha=1$.

Note. Since the case $\gamma=0$ is considered in [9], we only consider results for the case $\gamma>0$, except for Theorem 12 (see Remark 11).

Next, we introduce two known auxiliary functions [6]. Let

$$
\begin{gathered}
\phi_{\mu, \nu}(z)=1+\sum_{n=1}^{\infty} \frac{(n \nu+1)(n \mu+1)}{n+1} z^{n}, \\
\psi_{\mu, \nu}(z)=\phi_{\mu, \nu}^{-1}(z)=1+\sum_{n=1}^{\infty} \frac{n+1}{(n \nu+1)(n \mu+1)} z^{n} \\
=\iint_{0}^{1} \frac{d s d t}{\left(1-t^{\nu} s^{\mu} z\right)^{2}} .
\end{gathered}
$$

Here, $\phi_{\mu, \nu}^{-1}$ denotes the convolution inverse of $\phi_{\mu, \nu}$ such that $\phi_{\mu, v} * \phi_{\mu, \nu}^{-1}=1 /(1-z)$. By $*$, we mean the following: if $f$ and $g$ are in $\mathscr{A}$ with the power series expansions $f(z)=$ $\sum_{k=0}^{\infty} a_{k} z^{k}$ and $g(z)=\sum_{k=0}^{\infty} b_{k} z^{k}$, respectively, then the convolution or Hadamard product of $f$ and $g$ is given by $h(z)=$ $\sum_{k=0}^{\infty} a_{k} b_{k} z^{k}$

Since $v \geq 0, \mu \geq 0$, when $\gamma \geq 0$, making the change of variables $u=t^{\nu}, v=s^{\mu}$ in (7) results in writing $\psi_{\mu, \nu}$ as

$$
\psi_{\mu, \nu}(z)= \begin{cases}\frac{1}{\mu \nu} \iint_{0}^{1} \frac{u^{1 / \nu-1} v^{1 / \mu-1}}{(1-u v z)^{2}} d u d v, & \gamma>0, \\ \int_{0}^{1} \frac{d t}{\left(1-t^{\alpha} z\right)^{2}}, & \gamma=0, \alpha \geq 0 .\end{cases}
$$

Now, let $g$ be the solution of the initial value-problem

$$
\begin{aligned}
& \frac{d}{d t} t^{1 / \nu}(1+g(t)) \\
& \quad= \begin{cases}\frac{2}{\mu \nu} t^{1 / \nu-1} \int_{0}^{1} \frac{s^{1 / \mu-1}}{(1+s t)^{2}} d s, & \gamma>0, \\
\frac{2}{\alpha} \frac{t^{1 / \alpha-1}}{(1+t)^{2}}, & \gamma=0, \alpha>0,\end{cases}
\end{aligned}
$$

satisfying $g(0)=1$. The series solution is given by

$$
g(t)=2 \sum_{n=0}^{\infty} \frac{(n+1)(-1)^{n} t^{n}}{(1+\mu n)(1+\nu n)}-1
$$

Let $q$ be the solution of the differential equation

$$
\begin{aligned}
& \frac{d}{d t} t^{1 / \nu} q(t) \\
& \quad= \begin{cases}\frac{1}{\mu \nu} t^{1 / \nu-1} \int_{0}^{1} s^{1 / \mu-1} \frac{(1-s t)}{(1+s t)^{3}} d s, & \gamma>0, \\
\frac{1}{\alpha} t^{1 / \alpha-1} \frac{(1-t)}{(1+t)^{3}}, & \gamma=0, \alpha>0,\end{cases}
\end{aligned}
$$

satisfying $q(0)=0$. The series solution of $q(t)$ is given by

$$
q(t)=\sum_{n=0}^{\infty} \frac{(n+1)^{2}(-1)^{n} t^{n}}{(1+\mu n)(1+\nu n)}
$$

Note that $q(t)$ also satisfies $2 q(t)=t g^{\prime}(t)+g(t)+1$.

Our main result is the generalization of the following results given in $[6,7]$. The necessary and sufficient conditions under which the operator $V_{\lambda}(f(z))$ carries the function $f(z)$ from $W_{\beta}(\alpha, \gamma)$ to the classes $S^{*}$ and $\mathscr{C}$, respectively, are given in the next two results.

Theorem 1 (see [6]). Consider $\mu \geq 0, v \geq 0$ given by (5) and let $\beta<1$ satisfy

$$
\frac{\beta}{1-\beta}=-\int_{0}^{1} \lambda(t) g(t) d t
$$


where $g$ is the solution of the initial value-problem (9), and let $f \in W_{\beta}(\alpha, \gamma)$. Assume that $t^{1 / v} \Lambda_{\nu}(t) \rightarrow 0$, and $t^{1 / \mu} \Pi_{\mu, \nu}(t) \rightarrow$ 0 as $t \rightarrow 0^{+}$. Then, $F(z)=V_{\lambda}(f)(z)$ is in $S^{*}$ if and only if

$$
\begin{aligned}
& \operatorname{Re} \int_{0}^{1} \Pi_{\mu, \nu}(t) t^{1 / \mu-1}\left(\frac{h(t z)}{t z}-\frac{1}{(1+t)^{2}}\right) d t \geq 0, \quad \gamma>0, \\
& \operatorname{Re} \int_{0}^{1} \Pi_{0, \alpha}(t) t^{1 / \alpha-1}\left(\frac{h(t z)}{t z}-\frac{1}{(1+t)^{2}}\right) d t \geq 0, \quad \gamma=0,
\end{aligned}
$$

where

$$
\begin{gathered}
\Lambda_{v}(t)=\int_{t}^{1} \frac{\lambda(x)}{x^{1 / \nu}} d x, \quad v>0, \\
\Pi_{\mu, \nu}(t)= \begin{cases}\int_{t}^{1} \Lambda_{v}(x) x^{1 / v-1-1 / \mu} d x, & \gamma>0(\mu>0, \quad v>0), \\
\Lambda_{\alpha}(t), & \gamma=0(\mu=0, \quad v=\alpha>0),\end{cases} \\
h(z)=\frac{z(1+((\epsilon-1) / 2) z)}{(1-z)^{2}}, \quad|\epsilon|=1 .
\end{gathered}
$$

Theorem 2 (see [7]). Let $f \in W_{\beta}(\alpha, \gamma)$, let $\mu \geq 0, \nu \geq 0$ satisfy (5), and let $\beta<1$ be given by

$$
\frac{\beta-1 / 2}{1-\beta}=-\int_{0}^{1} \lambda(t) q(t) d t
$$

where $q$ is given by (11). Further, $\Lambda_{\nu}(t)$ and $\Pi_{\nu, \mu}(t)$ are given in (15) and (16), and assume that $t^{1 / \mu} \Lambda_{v}(t) \rightarrow 0$ and $t^{1 / v} \Pi_{\mu, \nu}(t) \rightarrow 0$ as $t \rightarrow 0^{+}$. Then

$$
\begin{aligned}
\operatorname{Re} \int_{0}^{1} \Pi_{\nu, \mu}(t) t^{1 / \mu-1}\left(h^{\prime}(t z)-\frac{1-t}{(1+t)^{3}}\right) d t & \geq 0, \\
\gamma & >0, \\
\operatorname{Re} \int_{0}^{1} \Pi_{0, \alpha}(t) t^{1 / \alpha-1}\left(h^{\prime}(t z)-\frac{1-t}{(1+t)^{3}}\right) d t & \geq 0, \\
\gamma & =0,
\end{aligned}
$$

if and only if $F(z)=V_{\lambda}(f)(z)$ is in $\mathscr{C}$.

It is difficult to verify conditions (14) and (19). Hence, the following results involving sufficient conditions are useful for finding applications.

Theorem 3 (see [6]). Let $\Lambda_{\nu}$ and $\Pi_{\mu, \nu}$ be defined in (15) and (16). Assume that both $\Pi_{\mu, \nu}$ and $\Lambda_{\nu}$ are integrable on $[0,1]$ and positive on $(0,1)$. Assume further that $\mu \geq 1$ and

$$
\frac{\Pi_{\mu, \nu}(t)}{1-t^{2}}
$$

is decreasing on $(0,1)$. If $\beta$ satisfy (13) and $f \in W_{\beta}(\alpha, \gamma)$, then $V_{\lambda}(f) \in S^{*}$.
Theorem 4 (see [7]). Let $\Lambda_{\nu}$ and $\Pi_{\mu, \nu}$ be defined in (15) and (16). Assume that both are integrable on $[0,1]$ and positive on $(0,1)$. Assume further that $\mu \geq 1$ and

$$
\frac{\Lambda_{v}(t) t^{1 / \nu-1 / \mu}+(1-1 / \mu) \Pi_{\mu, \nu}(t)}{1-t^{2}}
$$

is decreasing on $(0,1)$. If $\beta$ satisfy (18) and $f \in W_{\beta}(\alpha, \gamma)$, then $V_{\lambda}(f) \in \mathscr{C}$.

\section{Main Results}

We start with a result that gives both necessary and sufficient conditions for an integral transform that satisfies the admissibility property of the class $W_{\beta}(\alpha, \gamma)$, which contains nonunivalent functions also, to the Pascu class $M(\xi)$.

Theorem 5. Let $\mu>0, \nu>0$ satisfy (5), and let $\beta<1$ satisfy

$$
\frac{\beta}{(1-\beta)}=-\int_{0}^{1} \lambda(t)[(1-\xi) g(t)+\xi(2 q(t)-1)] d t
$$

where $g(t)$ and $q(t)$ are defined by the differential equations given in (9) and (11), respectively. Assume that $t^{1 / v} \Lambda_{\nu}(t) \rightarrow 0$ and $t^{1 / \mu} \Pi_{\mu, \nu}(t) \rightarrow 0$ as $t \rightarrow 0^{+}$. Then,

$$
N_{\Pi_{\mu, \nu}} \geq 0 \Longleftrightarrow F=V_{\lambda}\left(W_{\beta}(\alpha, \gamma)\right) \in M(\xi)
$$

or

$$
\xi z F^{\prime}+(1-\xi) F \in S^{*}
$$

where

$$
\begin{aligned}
N_{\Pi_{\mu, \nu}}(h)= & \inf _{z \in \Delta} \int_{0}^{1} t^{1 / \mu-1} \Pi_{\mu, \nu}(t) \mathscr{L}_{\xi, z}(t) d t, \\
\mathscr{L}_{\xi, z}(t)= & (1-\xi)\left(\operatorname{Re} \frac{h(t z)}{t z}-\frac{1}{(1+t)^{2}}\right) \\
& +\xi\left(\operatorname{Re} h^{\prime}(t z)-\frac{(1-t)}{(1+t)^{3}}\right) .
\end{aligned}
$$

The value of $\beta$ is sharp.

Proof. Let

$$
\begin{gathered}
H(z)=(1-\alpha+2 \gamma) \frac{f(z)}{z}+(\alpha-2 \gamma) f^{\prime}(z)+\gamma z f^{\prime \prime}(z), \\
G(z)=\frac{(H(z)-\beta)}{1-\beta} .
\end{gathered}
$$


Since $\operatorname{Re} e^{i \phi} G(z)>0$, [11] we may assume that $G(z)=(1+$ $x z) /(1+y z),|x|=|y|=1$. Further, assuming that $f(z)=$ $z+\sum_{n=2}^{\infty} a_{n} z^{n}$, from (5) we get

$$
\begin{aligned}
H(z)= & (1+\mu \nu-\nu-\mu) \frac{f(z)}{z}+(\nu+\mu-\mu \nu) f^{\prime}(z) \\
& +\mu \nu z f^{\prime \prime}(z) \\
= & \mu \nu z^{1-1 / \mu} \frac{d}{d z}\left[z^{1 / \mu-1 / v+1} \frac{d}{d z}\left(z^{1 / \nu-1} f(z)\right)\right] \\
= & f^{\prime}(z) * \phi_{\mu, \nu}(z) \\
\Longrightarrow & f^{\prime}(z)=H(z) * \psi_{\mu, \nu}=\left[(1-\beta)\left(\frac{1+x z}{1+y z}\right)+\beta\right] * \psi_{\mu, \nu},
\end{aligned}
$$

using (6) and (7). This gives

$$
\frac{f(z)}{z}=\frac{1}{z} \int_{0}^{z}\left[(1-\beta)\left(\frac{1+x \omega}{1+y \omega}\right)+\beta\right] d \omega * \psi_{\mu, v} .
$$

Since $F \in M(\xi)$ implies that $\xi z F^{\prime}+(1-\xi) F \in S^{*}$, by the well-known result [11, page 94] of convolution theory we get

$$
\begin{aligned}
& \xi z F^{\prime}+(1-\xi) F \in S^{*} \\
& \quad \text { iff } 0 \neq \frac{1}{z}\left[\left((1-\xi) F+\xi z F^{\prime}\right) * h(z)\right], \quad|z|<1 .
\end{aligned}
$$

Thus,

$$
\begin{aligned}
0 \neq & \left((1-\xi) \frac{F}{z}+\xi F^{\prime}\right) * \frac{h(z)}{z} \\
= & (1-\xi) \int_{0}^{1} \frac{\lambda(t)}{1-t z} * \frac{f(z)}{z} * \frac{h(z)}{z}+\xi \\
& \times \int_{0}^{1} \frac{\lambda(t)}{1-t z} * f^{\prime}(z) * \frac{h(z)}{z} \\
= & (1-\xi) \int_{0}^{1} \lambda(t) \frac{h(t z)}{t z} d t \\
& *\left[\frac{1}{z} \int_{0}^{z}(1-\beta)\left(\frac{1+x \omega}{1+y \omega}\right) d \omega+\beta\right] \\
& +\xi \int_{0}^{1} \lambda(t) h^{\prime}(t z) d t \\
& *\left[\frac{1}{z} \int_{0}^{z}(1-\beta)\left(\frac{1+x \omega}{1+y \omega}\right) d \omega+\beta\right] \\
= & (1-\beta)\left[(1-\xi)\left[\int_{0}^{1} \lambda(t)\left(\frac{1}{z} \int_{0}^{z} \frac{h(t \omega)}{t \omega} d \omega\right) d t+\frac{\beta}{1-\beta}\right]\right. \\
& * \psi(z) * \frac{1+x z}{1+y z} . \\
& \left.+\xi\left[\int_{0}^{1} \lambda(t)\left(\frac{1}{z} \int_{0}^{z} h^{\prime}(t \omega) d \omega\right) d t+\frac{\beta}{1-\beta}\right]\right]
\end{aligned}
$$

This condition holds if and only if [11, page 23]

$$
\begin{aligned}
& \operatorname{Re}((1-\beta) \\
& \times\left[(1-\xi)\left[\int_{0}^{1} \lambda(t)\left(\frac{1}{z} \int_{0}^{z} \frac{h(t \omega)}{t \omega} d \omega\right) d t+\frac{\beta}{1-\beta}\right]\right. \\
& \left.\quad+\xi\left[\int_{0}^{1} \lambda(t)\left(\frac{1}{z} \int_{0}^{z} h^{\prime}(t \omega) d \omega\right) d t+\frac{\beta}{1-\beta}\right]\right] \\
& * \psi(z))>\frac{1}{2} .
\end{aligned}
$$

Using the results (14) and (19) from Theorems 1 and 2, respectively, we obtain

$$
\begin{aligned}
& \int_{0}^{1} t^{1 / \mu-1} \Pi_{\mu, \nu}(t)[(1-\xi)\left(\operatorname{Re} \frac{h(t z)}{t z}-\frac{1}{(1+t)^{2}}\right) \\
&\left.+\xi\left(\operatorname{Re} h^{\prime}(t z)-\frac{(1-t)}{(1+t)^{3}}\right)\right] d t \geq 0
\end{aligned}
$$

which is the required result.

To verify sharpness, let $W_{\beta}(\alpha, \gamma)$ be the solution of the differential equation

$$
\begin{aligned}
(1 & -\alpha+2 \gamma) \frac{f(z)}{z}+(\alpha-2 \gamma) f^{\prime}(z)+\gamma z f^{\prime \prime}(z) \\
& =\beta+(1-\beta) \frac{1+z}{1-z}
\end{aligned}
$$

where $\beta<\beta_{0}$ satisfies (22). Further simplification using (10) and (12) gives

$$
\begin{array}{r}
\frac{\beta_{0}}{1-\beta_{0}}=-1-\left[(1-\xi) \sum_{n=1}^{\infty} \frac{2(n+1)(-1)^{n} \tau_{n}}{(1+\mu n)(1+\nu n)}\right. \\
\left.+\xi \sum_{n=1}^{\infty} \frac{2(n+1)^{2}(-1)^{n} \tau_{n}}{(1+\mu n)(1+\nu n)}\right],
\end{array}
$$

where $\tau_{n}=\int_{0}^{1} \lambda(t) t^{n} d t$.

Clearly, $F=V_{\lambda}(f(z)) \in M(\xi) \Rightarrow K(z):=\xi z F^{\prime}+(1-\xi) F \epsilon$ $S^{*}$. Using $f^{\prime}(z)=H(z) * \psi_{\mu, \nu}$ and the series expansion of $f(z)$, we get

$$
f(z)=z+\sum_{n=1}^{\infty} \frac{2(1-\beta)}{(n \mu+1)(n \nu+1)} z^{n+1} .
$$

This means that

$$
\begin{aligned}
F=V_{\lambda}(f(z)) & =z+\sum_{n=1}^{\infty} \frac{2(1-\beta)}{(n \mu+1)(n \nu+1)}\left(\int_{0}^{1} \lambda(t) t^{n} d t\right) z^{n+1} \\
& =z+\sum_{n=1}^{\infty} \frac{2(1-\beta) \tau_{n}}{(n \mu+1)(n \nu+1)} z^{n+1} .
\end{aligned}
$$


Using (37), a simple computation gives

$$
\begin{aligned}
K(z)= & (1-\xi)\left(z+\sum_{n=1}^{\infty} \frac{2(1-\beta) \tau_{n} z^{n+1}}{(n \mu+1)(n \nu+1)}\right) \\
& +\xi\left(z+\sum_{n=1}^{\infty} \frac{2(1-\beta)(n+1) \tau_{n} z^{n+1}}{(n \mu+1)(n \nu+1)}\right) .
\end{aligned}
$$

This means that

$$
\begin{aligned}
\left.z K^{\prime}(z)\right|_{z=-1}= & 1+\xi \sum_{n=1}^{\infty} \frac{2(1-\beta)(n+1)^{2} \tau_{n}(-1)^{n}}{(n \mu+1)(n \nu+1)} \\
& +(1-\xi) \sum_{n=1}^{\infty} \frac{2(1-\beta)(n+1) \tau_{n}(-1)^{n}}{(n \mu+1)(n \nu+1)} \\
= & 1-\frac{(1-\beta)}{1-\beta_{0}}<0 .
\end{aligned}
$$

Hence, $z K^{\prime}(z)=0$ for some $z \in \mathbb{D}$, so $K(z)$ is not even locally univalent in $\mathbb{D}$. This shows that the result is sharp for $\beta$.

Remark 6. This result generalizes various results known in this direction. For example, $\xi=0$ gives Theorem 1 [6, Theorem 3.1], and $\xi=1$ gives Theorem 2 [7, Theorem 3.1]. For other particular cases with $\xi=0$ or 1 , we refer to $[6,7]$ and the references therein.

Theorem 7. Let $\Pi_{\mu, v}$ and $\Lambda_{v}$ be defined as in (15) and (16), respectively, with both of them being integrable on $[0,1]$ and positive on $(0,1)$. Further, assume that $0 \leq \xi \leq 1, \mu \geq 1$, and

$$
\frac{\xi t^{1 / \xi-1 / \mu+1} d\left(t^{1 / \mu-1 / \xi} \Pi_{\mu, \nu}(t)\right)}{\left(1-t^{2}\right)}
$$

is increasing on $(0,1)$. Then, for $\beta<1$ satisfying (22), $V_{\lambda}\left(W_{\beta}(\alpha, \gamma)\right) \in M(\xi)$.

Proof. Consider the following:

$$
\begin{aligned}
N_{\Pi_{\mu, \nu}}(h)= & \operatorname{Re} \int_{0}^{1} t^{1 / \mu-1} \Pi_{\mu, \nu}(t) \\
\times & {\left[(1-\xi)\left(\frac{h(t z)}{t z}-\frac{1}{(1+t)^{2}}\right)\right.} \\
& \left.+\xi\left(h^{\prime}(t z)-\frac{(1-t)}{(1+t)^{3}}\right)\right] d t \\
= & \int_{0}^{1} t^{1 / \mu-1} \Pi_{\mu, \nu}(t) \\
\times & {\left[(1-\xi)\left(\frac{h(t z)}{t z}-\frac{1}{(1+t)^{2}}\right)\right.} \\
& \left.\quad+\xi \frac{d}{d t}\left(\frac{h(t z)}{z}-\frac{t}{(1+t)^{2}}\right)\right] d t .
\end{aligned}
$$

Integration by parts gives

$$
\begin{aligned}
N_{\Pi_{\mu, \nu}}(h)= & (1-\xi) \operatorname{Re} \int_{0}^{1} t^{1 / \mu-1} \Pi_{\mu, \nu}(t) \\
& \times\left(\frac{h(t z)}{t z}-\frac{1}{(1+t)^{2}}\right) d t \\
& -\xi \operatorname{Re} \int_{0}^{1} \frac{d}{d t}\left(t^{1 / \mu-1} \Pi_{\mu, \nu}(t)\right) \\
& \times\left(\frac{h(t z)}{z}-\frac{t}{(1+t)^{2}}\right) d t \\
= & \operatorname{Re} \int_{0}^{1}\left(\left[(1-\xi)-\xi\left(\frac{1}{\mu}-1\right)\right]\right. \\
\times & \left(\frac{h(t z)}{t z}-\frac{1}{(1+t)^{2}}\right) d t . \\
= & \left.\operatorname{Re} \int_{0}^{1} \frac{t^{1 / \mu-1}\left(\left(1-\frac{\xi}{\mu}\right) \Pi_{\mu, \nu}(t)-\xi t \Pi_{\mu, \nu}^{\prime}(t)\right)}{t z}-\frac{1}{(1+t)^{2}}\right) d t, \\
\times & \left.(t) t^{1 / \mu-1}-\xi \Pi_{\mu, \nu}^{\prime}(t) t^{1 / \mu}\right)
\end{aligned}
$$

by a simple computation. It is easy to see that, from Theorems 3 and $4, N_{\Pi_{\mu, v}}(h) \geq 0$ only when $\xi\left(\Pi_{\mu, v}(t) /\left(1-t^{2}\right)\right)+(1-\xi)$ $\left(\left(\Lambda_{\nu}(t) t^{1 / \nu-1 / \mu}+(1-1 / \mu) \Pi_{\mu, \nu}(t)\right) /\left(1-t^{2}\right)\right)$ is decreasing on $(0,1)$ which is nothing but $(40)$, and the proof is complete by applying Theorem 5 .

Remark 8. Even though we did not consider the case $\gamma=$ 0 , even at $\gamma=0$, Theorem 7 does not reduce to a similar result given in [9]. This is due to the fact that our condition (40) has the term $\left(1-t^{2}\right)$ in the denominator, whereas the corresponding result in [9] has the term $\log (1 / t)$ and hence has different condition.

\section{Applications}

It is difficult to check the condition given in Section 2, for $V_{\lambda}\left(W_{\beta}(\alpha, \gamma)\right) \subset M(\xi)$. In order to find applications, simplified conditions are required. For this purpose, from (40), it is enough to show that

$$
\frac{\xi t^{1 / \xi-1 / \mu+1} d\left(t^{1 / \mu-1 / \xi} \Pi_{\mu, \nu}(t)\right)}{\left(1-t^{2}\right)}
$$

is increasing on $(0,1)$ which is equivalent to having the fact that

$$
g(t)=\frac{(1-\xi / \mu) \Pi_{\mu, \nu}(t)+\xi t^{(1 / v)-(1 / \mu)} \Lambda_{\nu}(t)}{\left(1-t^{2}\right)}
$$

is decreasing on $(0,1)$, where $\Lambda_{\nu}(t)$ and $\Pi_{\mu, \nu}(t)$ are defined in (15) and (16). It is enough to have $g^{\prime}(t) \leq 0$. 
Let $g(t)=p(t) /\left(1-t^{2}\right)$, where $p(t)=(1-\xi / \mu) \Pi_{\mu, \nu}(t)+$ $\xi t^{1 / \nu-1 / \mu} \Lambda_{\nu}(t)$. So to satisfy the above condition, we need to have

$$
L(t)=p(t)+\frac{\left(1-t^{2}\right) p^{\prime}(t)}{2 t} \leq 0 .
$$

Since $\Lambda_{\nu}(1)=0$ and $\Pi_{\mu, \nu}(1)=0$, we get $L(1)=0$. This implies that it suffices to have that $L(t)$ is increasing on $(0,1)$, which means that

$$
L^{\prime}(t)=\frac{\left(1-t^{2}\right)}{2 t^{2}}\left[t p^{\prime \prime}(z)-p^{\prime}(z)\right] \geq 0 .
$$

The above equation also holds if $t p^{\prime \prime}(z)-p^{\prime}(z) \geq 0$ which is equivalent to the condition

$$
\begin{gathered}
\left(\frac{\xi}{\nu}-1\right)\left(\frac{1}{\nu}-\frac{1}{\mu}-2\right) \Lambda_{\nu}(t)+\left(1+\xi\left[1+\frac{1}{\mu}-\frac{1}{\nu}\right]\right) \\
\times t^{1-1 / v} \lambda(t)-\xi t^{2-1 / v} \lambda^{\prime}(t) \geq 0 .
\end{gathered}
$$

This inequality can further be reduced to

$$
(1-\xi)\left[\left(1+\frac{1}{\mu}\right) \lambda(t)-t \lambda^{\prime}(t)\right]+\xi\left[t^{2} \lambda^{\prime \prime}(t)-\frac{1}{\mu} t \lambda^{\prime}(t)\right] \geq 0
$$

if the inequality

$$
\xi \frac{\lambda^{\prime}(1)}{\lambda(1)} \leq 1+\xi\left(1+\frac{1}{\mu}-\frac{1}{\nu}\right)
$$

is true. So, in order to obtain further results, we check conditions (48) and (49). Note that, whenever $\lambda(1)=\lambda^{\prime}(1)=0$, from (47) we see that it is sufficient to check (48) as there is no necessity for the condition given by (49).

As the first application, we consider $\lambda(t)=(c+1) t^{c}, c>$ -1 , so that the Bernardi operator of the function in $W_{\beta}(\alpha, \gamma)$ is in $M(\xi)$.

Theorem 9. Let $0 \leq \xi \leq 1, \nu \geq \mu \geq 1$, and let $\beta<1$ satisfy (22). If $f(z) \in W_{\beta}(\alpha, \gamma)$, then the function, given by the Bernardi operator,

$$
V_{\lambda}(f)(z)=(1+c) \int_{0}^{1} t^{c-1} f(t z) d t
$$

belongs to the class $M(\xi)$ if

$$
-1<c \leq \min \left[\left(1+\frac{1}{\mu}-\frac{1}{\nu}\right),\left(\frac{1+1 / \mu-\xi}{1+2 \xi}\right)\right] .
$$

Proof. With $\lambda(t)=(1+c) t^{c}$, using (51), we get

$$
\xi \frac{\lambda^{\prime}(1)}{\lambda(1)}=\xi c \leq \xi\left(1+\frac{1}{\mu}-\frac{1}{\nu}\right) \leq 1+\xi\left(1+\frac{1}{\mu}-\frac{1}{\nu}\right) .
$$

So, inequality (49) is satisfied. If inequality (48) holds, then $V_{\lambda}(f)(z) \in M(\xi)$, which means that

$$
\begin{aligned}
& (1-\xi)\left[\left(1+\frac{1}{\mu}\right)(c+1)-c(c+1)\right] \\
& \quad+\xi\left[(c-1) c(c+1)-\frac{1}{\mu} c(c+1)\right] \geq 0 .
\end{aligned}
$$

On further simplification, this inequality reduces to

$$
1+\frac{1}{\mu}-c+c^{2} \xi \geq 0
$$

Clearly, $(c+1)^{2}>0$. Hence, using $c^{2}>-(2 c+1)$ and substituting in (54), we get

$$
\left(c^{2} \xi+1+\frac{1}{\mu}-c\right)>\left(-c(2 \xi+1)+1+\frac{1}{\mu}-\xi\right),
$$

which is true by the hypothesis.

Remark 10. (1) When $\xi=0$, then $-1<c \leq \min [(1+1 / \mu-$ $1 / \nu),(1+1 / \mu)]=(1+1 / \mu-1 / \nu)$, whereas, in [6], the range for $c$ is given as $-1<c \leq(1+1 / \mu)$.

(2) For $\xi=1$, we have $-1<c \leq \min [(1+1 / \mu-1 / \nu)$, $(1 / 3 \mu)]=(1 / 3 \mu)$. The result obtained in [7], for $\nu \geq \mu \geq 1$, is $-1<c \leq(2+1 / \mu-1 / \nu)$.

So in both cases the result (Theorem 9) obtained for admissibility property of Bernardi operator in $M(\xi)$ class differs from [6, Theorem 5.1] and [7, Theorem 5.1]. But our result is true for $0<\xi<1$ also.

Remark 11. We recall that, throughout this paper, we use $\gamma>0$, as $\gamma=0$ case is considered in [9]. But we note that, for the Bernardi operator, the result given in [9] uses the fact $\lambda(1)=0$, which is not true. Hence, in order to make completion of the work for $W_{\beta}(\alpha, \gamma)$ in this direction, we give the result related to Bernardi operator for $\gamma=0$. Since the condition for $\lambda(t)$ given in [9] is different from the one given by (49) and (48), we explicitly prove this result.

Theorem 12. Let $0<\xi<1, \mu \geq 1$, and let $\beta<1$ satisfy (22). If $f(z) \in W_{\beta}(\alpha, \gamma)$, then the function

$$
V_{\lambda}(f)(z)=(1+c) \int_{0}^{1} t^{c-1} f(t z) d t
$$

belongs to the class $M(\xi)$ if $c>1+1 / \alpha$ and $\xi \geq \alpha$ for $\gamma=0$.

Proof. Note that, for the Bernardi operator, the case $\xi=0$ is considered in [6, Theorem 5.1] and $\xi=1$ is considered in [7, Theorem 5.1]. We consider only the case $0<\xi<1$.

For the case $\gamma=0$, the integral operator $V_{\lambda}\left(W_{\beta}(\alpha, \gamma)\right)$ is in $M(\xi)$ if and only if

$$
\frac{(1-\xi / \alpha) \Pi_{0, \alpha}(t)+\xi t^{1-(1 / \alpha)} \lambda(t)}{\left(1-t^{2}\right)} \text { is decreasing on }(0,1)
$$

which can be easily obtained as in Theorem 7 .

Let $J(t)=p(t) /\left(1-t^{2}\right)$, where

$$
p(t)=\left(1-\frac{\xi}{\alpha}\right) \Pi_{0, \alpha}(t)+\xi t^{1-1 / \alpha} \lambda(t)
$$

for the integral operator to be in $M(\xi), J^{\prime}(t) \leq 0 \Rightarrow p(t)+$ $\left(\left(1-t^{2}\right) / 2 t\right) p^{\prime}(t) \leq 0$. 
Substituting the value of $p(t)$ and $p^{\prime}(t)$ in the above equation, we have

$$
\begin{aligned}
J(t)= & \left(1-\frac{\xi}{\alpha}\right) \Pi_{0, \alpha}(t)+\frac{(\xi+1)}{2} t^{1-1 / \alpha} \lambda(t) \\
& +\frac{(\xi-1)}{2} t^{-1-1 / \alpha} \lambda(t)+\frac{\xi}{2} t^{-1 / \alpha}\left(1-t^{2}\right) \lambda^{\prime}(t) \leq 0 .
\end{aligned}
$$

Consider the case when $\lambda(t)=(c+1) t^{c}$, where $c>-1$. So inequality (59) on simplification reduces to

$$
\begin{aligned}
J(t)= & \left(1-\frac{\xi}{\alpha}\right) \frac{c+1}{c-1 / \alpha+1}\left[1-t^{c-1 / \alpha+1}\right] \\
& +\frac{1}{2}(c+1)(\xi+1-c \xi) t^{c+1-1 / \alpha} \\
& +\frac{1}{2}(c+1)(\xi-1+c \xi) t^{c-1-1 / \alpha} \leq 0 .
\end{aligned}
$$

If $c>1+1 / \alpha$ and $\alpha \leq \xi$, then, for $t=0,(60)$ is satisfied.

For $c>1+1 / \alpha, J(0) \leq 0$, which clearly implies that if $J(t)$ is decreasing on $t \in(0,1)$, that is, $J^{\prime}(t) \leq 0$, then also inequality (60) holds. Further simplifying (59), for Bernardi operator, we need to show that

$$
\begin{aligned}
\frac{1}{2}(1-\xi) & \left(1+\frac{1}{\alpha}\right) \lambda(t)+\frac{1}{2}\left[\xi\left(1-\frac{1}{\alpha}\right)-1\right] t \lambda^{\prime}(t) \\
+ & \frac{\xi}{2} t^{2} \lambda^{\prime \prime}(t) \leq 0 .
\end{aligned}
$$

On substituting the values of $\lambda(t), \lambda^{\prime}(t)$, and $\lambda^{\prime \prime}(t)$ in (61), we get

$$
\left(1-c-\frac{1}{\alpha}\right)-\xi\left(1-c^{2}-\frac{1}{\alpha}+\frac{c}{\alpha}\right) \leq 0 .
$$

To satisfy (62), $\xi$ should hold:

$$
\xi \geq \frac{\alpha(1-c)-1}{(c-1)+\alpha\left(1-c^{2}\right)} .
$$

Since $\alpha \leq \xi$, we get

$$
\xi \geq \max \left(\alpha, \frac{\alpha(1-c)-1}{(c-1)+\alpha\left(1-c^{2}\right)}\right)=\alpha,
$$

satisfying the hypothesis of the theorem which gives that $V_{\lambda}(f)(z)$ given by $(56)$ is in $M(\xi)$.

Theorem 13. Let $0 \leq \xi \leq 1$ and $\nu \geq \mu \geq 1$. If $F \in \mathscr{A}$ satisfies

$$
\operatorname{Re}\left(F^{\prime}(z)+\alpha z F^{\prime \prime}(z)+\gamma z^{2} F^{\prime \prime \prime}(z)\right)>\beta
$$

in $\mathbb{D}$ and let $\beta<1$ satisfy

$$
\frac{\beta}{(1-\beta)}=-\int_{0}^{1} \lambda(t)[(1-\xi) g(t)+\xi(2 q(t)-1)] d t,
$$

where $g(t)$ and $q(t)$ are given by (10) and (12), respectively, then $F \in M(\xi)$.
Proof. Let $f(z)=z F^{\prime}(z)$; then, $f \in W_{\beta}(\alpha, \gamma)$. Therefore,

$$
F(z)=\int_{0}^{1} \frac{f(t z)}{t} d t
$$

When $c=0$, the hypothesis $v \geq \mu \geq 1$ satisfies (51), and, hence, from Theorem 9, the required result follows.

Example 14. If $\gamma=1, \alpha=3$, then $\mu=1=\nu$. In this case, (10) and (12) yield

$$
\begin{aligned}
\frac{\beta}{(1-\beta)} & =2(1-\xi) \int_{0}^{-1} \frac{\log (1-t)}{t} d t-2 \xi \log 2+1 \\
& =1-2(1-\xi)\left(\frac{\pi^{2}}{12}\right)-2 \xi \log 2 .
\end{aligned}
$$

Thus, $\operatorname{Re}\left(f^{\prime}(z)+3 z f^{\prime \prime}(z)+z^{2} f^{\prime \prime \prime}(z)\right)>\beta \Rightarrow f \in M(\xi)$.

Remark 15. (1) For $\xi=0[6$, Remark 5.2], we get $\beta=$ -1.816378 , such that $f \in M(0)$.

(2) For $\xi=1$ [7, Example 5.2], we get $\beta=-0.629445$, such that $f \in M(1)$.

Theorem 16. Let $0 \leq \xi \leq 1, \mu \geq 1, A>0, C>0$, and $0<B<1$, and let $\beta<1$ satisfy (18). If $f(z) \in W_{\beta}(\alpha, \gamma)$, then the function

$$
V_{\lambda}(f)(z)=k \int_{0}^{1} t^{B-1}(1-t)^{C-A-B} \phi(1-t) \frac{f(t z)}{t} d t
$$

belongs to the class $M(\xi)$ if

$$
B<\min \left(\left[2+\frac{1}{\mu}\right],(C-A-1)\right) .
$$

Proof. For $V_{\lambda}(f)(z)$ to be in $M(\xi), \lambda(t)$ should satisfy (47), where

$$
\begin{aligned}
\lambda(t)= & k t^{B-1}(1-t)^{C-A-B} \phi(1-t), \\
\lambda^{\prime}(t)= & K t^{B-2}(1-t)^{C-A-B-1} \\
& \times[((B-1)(1-t)-(C-A-B) t) \phi(1-t) \\
& \left.\quad-t(1-t) \phi^{\prime}(1-t)\right],
\end{aligned}
$$

$$
\begin{aligned}
& \lambda^{\prime \prime}(t)=K t^{B-3}(1-t)^{C-A-B-2} \\
& \times[\left((B-1)(B-2)(1-t)^{2}\right. \\
&-2(B-1)(C-A-B) t(1-t)+(C-A-B) \\
&\left.\times(C-A-B-1) t^{2}\right) \phi(1-t) \\
&+[2(C-A-B) t-2(B-1)(1-t)] \\
&\left.\times t(1-t) \phi^{\prime}(1-t)+t^{2}(1-t)^{2} \phi^{\prime \prime}(1-t)\right] .
\end{aligned}
$$


Since $C-A-B>1, \lambda(1)=0$ and $\lambda^{\prime}(1)=0$ hold. Hence it is enough to check (48). If we substitute the values of $\lambda(t)$, $\lambda^{\prime}(t)$, and $\lambda^{\prime \prime}(t)$ in (48), a simple computation shows that (48) is equivalent to

$$
(1-\xi) h_{1}(t)+\xi h_{2}(t) \geq 0,
$$

where

$$
\begin{aligned}
h_{1}(t)= & k t^{B-1}(1-t)^{C-A-B-1} \\
& \times\left(X_{1}(t) \phi(1-t)-t(1-t) \phi^{\prime}(1-t)\right)
\end{aligned}
$$

with

$$
\begin{aligned}
X_{1}(t)= & {\left[\left(1+\frac{1}{\mu}\right)(1-t)-(B-1)(1-t)+(C-A-B) t\right], } \\
h_{2}(t)= & k t^{B-1}(1-t)^{C-A-B-2} \\
& \times\left(X_{2}(t) \phi(1-t)+X_{3}(t) \phi^{\prime}(1-t)+X_{4}(t) \phi^{\prime \prime}(1-t)\right)
\end{aligned}
$$

with

$$
\begin{aligned}
X_{2}(t)= & (B-1)(1-t)^{2}\left[B-2-\frac{1}{\mu}\right] \\
& +(C-A-B) t(1-t)\left[\frac{1}{\mu}-2(B-1)\right] \\
& +(C-A-B)(C-A-B-1) t^{2}, \\
X_{3}(t)= & {[2(C-A-B) t-2(B-1)(1-t)] } \\
& \times t(1-t)+\frac{1}{\mu} t(1-t)^{2}, \\
X_{4}(t)= & t^{2}(1-t)^{2} .
\end{aligned}
$$

Since $\phi(1-t)>0, \phi^{\prime}(1-t)>0$, and $\phi^{\prime \prime}(1-t)>0$ (see also $[6,7,9])$, proving $X_{i}(t)>0$ for $i=1,2,3$ gives $h_{1}(t) \geq 0$ and $h_{2}(t) \geq 0$ which will imply the required result for $0<t<1$.

Now, $X_{1}(t)=[(C-A-B) t+(1+1 / \mu-(B-1))(1-t)]>0$, which clearly holds since $(C-A-B)>1$ and $B<2+1 / \mu$ for all $t \in(0,1)$.

Similarly, to prove $X_{2}(t)>0$, it is enough to prove

$$
\begin{aligned}
& (B-1)\left(B-2-\frac{1}{\mu}\right)(1-t)+(C-A-B) \\
& \quad \times\left(\frac{1}{\mu}-2(B-1)\right) t>0,
\end{aligned}
$$

as the other term in $X_{2}(t)$ is positive on $0<t<1$. Since $B<1$ and $1 / \mu>(B-2)$ from the hypothesis, the term involving $(1-t)$ is nonnegative. Given that $1 / \mu>(B-2)$, since $B<1$, we have $1 / \mu>2(B-1)$ and $(C-A-B)>1$, which gives $X_{2}(t)>0$ for $0<t<1$.

Now, proving that $X_{3}(t)>0$ is equivalent to proving that $2(C-A-B) t+[1 / \mu-2(B-1)](1-t)>0$.

Since $2(C-A-B) t+[1 / \mu-2(B-1)](1-t)>0$, by hypothesis, and $(1 / \mu) t(1-t)^{2}>0$ for $\mu>0$ and $0<t<1$, $X_{3}(t)>0$.
Remark 17. (1) For the particular value of $\xi=0$, Theorem 16 yields a result with a smaller range for the parameters than the result given in [6, Theorem 5.5].

(2) For the case $\xi=1$, the result of Theorem 16 coincides with the result given in [7, Theorem 5.8]

Theorem 18. Let $0 \leq \xi \leq 1, a>-1$, and $b>-1$, and let $\beta<1$ satisfy (18). If $f(z) \in W_{\beta}(\alpha, \gamma)$, then the function

$$
V_{\lambda}(f)(z)=\int_{0}^{1} \lambda(t) \frac{f(t z)}{t} d t
$$

where $\lambda(t)$ is given by

$$
\lambda(t)= \begin{cases}(a+1)(b+1) \frac{t^{a}\left(1-t^{b-a}\right)}{b-a}, & b \neq a, \\ (a+1)^{2} t^{a} \log \left(\frac{1}{t}\right), & b=a,\end{cases}
$$

belongs to the class $M(\xi)$ if $a, b$, and $\mu$ satisfy one of the following conditions:

(i) $b>a,-1<a<0$, and $b+a-1<1 / \mu<b-1$,

(ii) $b<a,-1<b<0$, and $b+a-1<1 / \mu<a-1$,

(iii) $b=a<0$ and $1 / \mu>b-1$, which for $\mu \geq 1$ (as in Theorem 7) gives $b<2 \Rightarrow b<\min \{0,2\}=0$.

Proof. To prove the required result, we need to show that (47) holds.

For the case $a \neq b, \lambda(1)=0, \Lambda_{\nu}(1)=0$, and $\lambda^{\prime}(t) \leq 0$. So, it is enough to show inequality (48). On substituting the values for $\lambda(t), \lambda^{\prime}(t)$, and $\lambda^{\prime \prime}(t)$, we need to show that (1 $\xi) A(t)+\xi B(t) \geq 0$, where

$$
\begin{aligned}
A(t)= & \frac{(a+1)(b+1)}{(b-a)} \\
& \times\left(\left[\left(1+\frac{1}{\mu}\right)-a\right] t^{a}-\left[\left(1+\frac{1}{\mu}\right)-b\right] t^{b}\right), \\
B(t)= & \frac{(a+1)(b+1)}{(b-a)} \\
& \times\left(\left[a(a-1)-\frac{1}{\mu} a\right] t^{a}-\left[b(b-1)-\frac{1}{\mu} b\right] t^{b}\right) .
\end{aligned}
$$

So, it is enough to prove $A(t)>0$ and $B(t)>0$ for $0<t<1$.

Case $i(a<b)$. Since $a>-1$ and $b>-1$, so $(a+1)(b+$ $1) /(b-a)>0$. We need only to show that $[(1+1 / \mu)-a] t^{a}-$ $[(1+1 / \mu)-b] t^{b}>0$, which clearly holds since $b>(1+1 / \mu)$. Hence, $A(t)>0$ for all $t \in(0,1)$. Now, for $B(t)$ to be positive, it is enough to show that $\left([a(a-1)-(1 / \mu) a] t^{a}-[b(b-1)-\right.$ $\left.(1 / \mu) b] t^{b}\right)>0$, which is satisfied by the given condition on $b$.

Case ii $(b<a)$. Since $a>-1$ and $b>-1$, so $(a+1)(b+1) /(b-$ $a)<0$. We need only to show that $[(1+1 / \mu)-a] t^{a}-[(1+$ $1 / \mu)-b] t^{b}<0$, which is true since $a>(1+1 / \mu)$. Now, for $B(t)$ to be positive, it is enough to show that

$$
\left(\left[a(a-1)-\frac{1}{\mu} a\right] t^{a}-\left[b(b-1)-\frac{1}{\mu} b\right] t^{b}\right)<0,
$$

which is satisfied by the given condition on $a$. 
Case iii ( $a=b \leq 0)$. We change inequality (48), which is true for $a=0$. Hence, we only consider the situation $a<0$. Substituting the values of $\lambda(t), \lambda^{\prime}(t)$, and $\lambda^{\prime \prime}(t)$ in (48), an easy computation shows that, for $0 \leq \xi \leq 1$, it suffices to show that the expressions

$$
\begin{gathered}
\log \left(\frac{1}{t}\right)\left(1+\frac{1}{\mu}-a\right)+1 \\
\left(a(a-1)-\frac{a}{\mu}\right) \log \left(\frac{1}{t}\right)+\left(\frac{1}{\mu}-2 a+1\right)
\end{gathered}
$$

are nonnegative. Since $\log (1 / t)$ is positive, the nonnegativity of the first expression $\log (1 / t)(1+1 / \mu-a)+1$ follows from hypothesis (iii) of the theorem. Similar observation shows that the second expression reduces to $a(a-1)-a / \mu \geq 0$ and $a / \mu-2 a+1 \geq 0$ using $\log (1 / t)$ is positive. These two inequalities, for $a<0$, give $1 / \mu \geq \max \{2 a-1, a-1\}=$ $a-1$, which is hypothesis (iii). The proof of Theorem 18 is completed.

Theorem 19. Let $c<0$ and $\mu \geq 1$ with $0 \leq \xi \leq 1$. Further, suppose that $p>2$ and let $\beta<1$ satisfy

$$
\frac{\beta-1 / 2}{1-\beta}=-\frac{(1+c)^{p}}{\Gamma(p)} \int_{0}^{1} t^{c}\left(\log \frac{1}{t}\right)^{p-1} q(t) d t
$$

where $q(t)$ satisfies (11). Then, for the function $f(z) \in W_{\beta}(\alpha, \gamma)$,

$$
V_{\lambda}(f)=\frac{(c+1)^{p}}{\Gamma(p)} \int_{0}^{1}\left(\log \frac{1}{\gamma}\right)^{p-1} t^{c-1} f(t z) d t
$$

belongs to the class $M(\xi)$.

Proof. Choosing $\phi(1-t)=((\log (1 / t)) /(1-t))^{p-1}$, we take $C-A-B=p-1$ and $B=c+1$ so that $\lambda(t)$ takes the form

$$
\lambda(t)=K t^{c}(1-t)^{p-1} \phi(1-t), \quad K=\frac{(1+c)^{p}}{\Gamma(p)} .
$$

We complete the proof by applying Theorem 16 and using a simple computation to obtain $c<\min \{0,1+1 / \mu\}=0$.

\section{A Generalized Integral Operator}

In this section, for the functions $f \in W_{\beta}(\alpha, \gamma)$, we consider another integral operator introduced in [5] and find the admissibility conditions to be in the class $M(\xi)$.

Theorem 20. Let $\mu>0, \nu>0$ satisfy (5); let $\rho<1$, and $\beta<1$ satisfies

$$
\begin{aligned}
& \frac{1}{2(1-\beta)(1-\rho)} \\
& \quad=\int_{0}^{1} \lambda(t)\left[(1-\xi)\left(\frac{1-g(t)}{2}\right)+\xi(1-q(t))\right] d t
\end{aligned}
$$

where $g(t)$ and $q(t)$ are given in (10) and (12), respectively. Assume that, for $f \in \mathscr{A}$,

$$
\mathscr{V}_{\lambda}(f)(z)=z \int_{0}^{1} \lambda(t)\left(\frac{1-\rho t z}{1-t z}\right) d t * f(z) .
$$

Then, $F=\mathscr{V}_{\lambda}\left(W_{\beta}(\alpha, \gamma)\right) \subset M(\xi) \Leftrightarrow N_{\Pi_{\mu, \nu}}(h) \geq 0$.

Proof. The proof follows similar lines of the proof of Theorem 5. Hence, we omit the details.

Note. $\mathscr{V}_{\lambda}(f)(z)=\rho z+(1-\rho) V_{\lambda}(f)(z)$ and, hence, this operator generalizes the operator given in (3).

For finding applications of the operator $\mathscr{V}_{\lambda}(f)(z)$, by virtue of (25), Theorem 12 is sufficient. This means that we use the conditions given in Section 3. Hence, we state the following results without giving their proofs as they can be obtained in a similar fashion as in the results of Section 3.

Corollary 21. Let $0 \leq \xi \leq 1, \nu>1$, and $\mu>1$ satisfy (22). Let $\rho<1$, and $\beta<1$ satisfies

$$
\begin{aligned}
& \frac{1}{2(1-\beta)(1-\rho)} \\
& \quad=(c+1) \int_{0}^{1} t^{c}\left[(1-\xi)\left(\frac{1-g(t)}{2}\right)+\xi(1-q(t))\right] d t
\end{aligned}
$$

where $g(t)$ and $q(t)$ are given by (10) and (12), respectively. Assume that, for $f \in W_{\beta}(\alpha, \gamma)$, the function $\mathscr{V}_{\lambda}(f)(z)$ belongs to the class $M(\xi)$ provided

$$
-1<c \leq \min \left[\left(1+\frac{1}{\mu}-\frac{1}{\nu}\right),\left(\frac{1+\mu(1-\xi)}{\mu(1+2 \xi)}\right)\right] .
$$

Corollary 22. Let $0 \leq \xi \leq 1, \nu>1$, and $\mu>1$ satisfy (22). Let $\rho<1$, and $\beta<1$ satisfies

$$
\begin{aligned}
& \frac{1}{2(1-\beta)(1-\rho)} \\
& =k \int_{0}^{1} t^{B-1}(1-t)^{C-A-B} \phi(1-t) \\
& \quad \times\left[(1-\xi)\left(\frac{1-g(t)}{2}\right)+\xi(1-q(t))\right] d t,
\end{aligned}
$$

where $g(t)$ and $q(t)$ are given by (10) and (12), respectively. Assume that, for $f \in W_{\beta}(\alpha, \gamma)$, the function $\mathscr{V}_{\lambda}(f)(z)$ belongs to the class $M(\xi)$ provided

$$
B<\min \left(\left[2+\frac{1}{\mu}\right],(C-A-1)\right) .
$$

Corollary 23. Let $0 \leq \xi \leq 1, a>-1$, and $b>-1$. Let $\rho<1$, and $\beta<1$ satisfies

$$
\begin{aligned}
& \frac{1}{2(1-\beta)(1-\rho)} \\
& \quad=\int_{0}^{1} \lambda(t)\left[(1-\xi)\left(\frac{1-g(t)}{2}\right)+\xi(1-q(t))\right] d t
\end{aligned}
$$


where $g(t)$ and $q(t)$ are given by (10) and (12), respectively, and $\lambda(t)$ is given by

$$
\lambda(t)= \begin{cases}(a+1)(b+1) \frac{t^{a}\left(1-t^{b-a}\right)}{b-a}, & b \neq a, \\ (a+1)^{2} t^{a} \log \left(\frac{1}{t}\right), & b=a .\end{cases}
$$

Assume that, for $f \in W_{\beta}(\alpha, \gamma)$, the function $\mathscr{V}_{\lambda}(f)(z)$ belongs to the class $M(\xi)$ provided

(i) $b>a,-1<a<0$, and $b+a-1<1 / \mu<b-1$,

(ii) $b<a,-1<b<0$, and $b+a-1<1 / \mu<a-1$,

(iii) $b=a<0$ and $1 / \mu>b-1$, which for $\mu \geq 1$ (as in Theorem 7) gives $b<2 \Rightarrow b<\min \{0,2\}=0$.

Corollary 24. Let $c<0, \mu \geq 1$ satisfy (22), with $0 \leq \xi \leq 1$. Let $p>2, \rho<1$, and $\beta<1$ satisfies

$$
\begin{aligned}
& \frac{1}{2(1-\beta)(1-\rho)} \\
& =\frac{(1+c)^{p}}{\Gamma(p)} \int_{0}^{1} t^{c}\left(\log \frac{1}{t}\right)^{p-1} \\
& \times\left[(1-\xi)\left(\frac{1-g(t)}{2}\right)+\xi(1-q(t))\right] d t
\end{aligned}
$$

where $g(t)$ and $q(t)$ are given by (10) and (12), respectively. Then, for $f \in W_{\beta}(\alpha, \gamma)$ the function $\mathscr{V}_{\lambda}(f)(z)$ belongs to the class $M(\xi)$.

Remark 25. All the above applications at $\rho=0$ reduce to the results obtained in Section 3. Further, Corollary 21 at $\xi=1$ and $\xi=0$ reduces, respectively, to Corollaries 6.4 and 6.5 given in [7]. All the other corollaries in this section for $\rho \neq 0$ and $0<\xi<1$ are not discussed in the literature elsewhere.

\section{Conflict of Interests}

The authors declare that there is no conflict of interests regarding the publication of this paper.

\section{References}

[1] P. L. Duren, Univalent Functions, vol. 259 of Grundlehren der Mathematischen Wissenschaften, Springer, New York, NY, USA, 1983.

[2] N. N. Pascu and V. Podaru, "On the radius of alpha-starlikeness for starlike functions of order beta," in Complex Analysis-Fifth Romanian-Finnish Seminar, Part 1 (Bucharest, 1981), vol. 1013 of Lecture Notes in Mathematics, pp. 336-349, Springer, Berlin, Germany, 1983.

[3] R. Fournier and S. Ruscheweyh, "On two extremal problems related to univalent functions," The Rocky Mountain Journal of Mathematics, vol. 24, no. 2, pp. 529-538, 1994.

[4] Y. C. Kim and F. Rønning, "Integral transforms of certain subclasses of analytic functions," Journal of Mathematical Analysis and Applications, vol. 258, no. 2, pp. 466-489, 2001.
[5] R. M. Ali and V. Singh, "Convexity and starlikeness of functions defined by a class of integral operators," Complex Variables. Theory and Application, vol. 26, no. 4, pp. 299-309, 1995.

[6] R. M. Ali, A. O. Badghaish, V. Ravichandran, and A. Swaminathan, "Starlikeness of integral transforms and duality," Journal of Mathematical Analysis and Applications, vol. 385, no. 2, pp. 808-822, 2012.

[7] R. M. Ali, M. M. Nargesi, and V. Ravichandran, "Convexity of integral transforms and duality," Complex Variables and Elliptic Equations, vol. 58, no. 11, pp. 1569-1590, 2013.

[8] S. Verma, S. Gupta, and S. Singh, "On an integral transform of a class of analytic functions," Abstract and Applied Analysis, vol. 2012, Article ID 259054, 10 pages, 2012.

[9] K. Raghavendar and A. Swaminathan, "Integral transforms of functions to be in certain class defined by the combination of starlike and convex functions," Computers \& Mathematics with Applications, vol. 63, no. 8, pp. 1296-1304, 2012.

[10] R. M. Ali, S. K. Lee, K. G. Subramanian, and A. Swaminathan, "A third-order differential equation and starlikeness of a double integral operator," Abstract and Applied Analysis, vol. 2011, Article ID 901235, 10 pages, 2011.

[11] S. Ruscheweyh, Convolutions in Geometric Function Theory, vol. 83 of Séminaire de Mathématiques Supérieures, Presses de l'Université de Montréal, Montreal, Canada, 1982. 


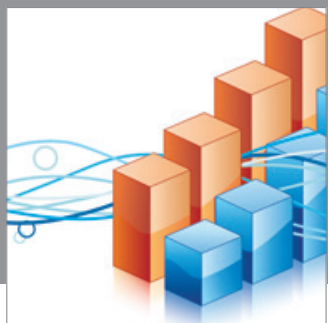

Advances in

Operations Research

mansans

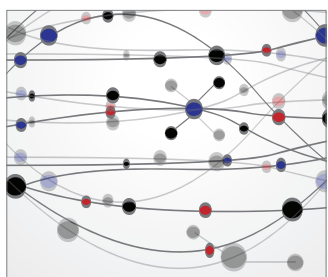

The Scientific World Journal
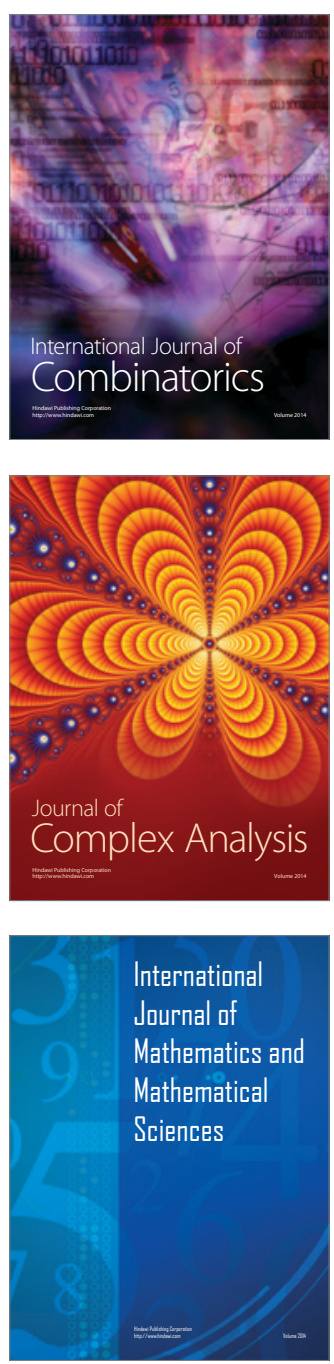
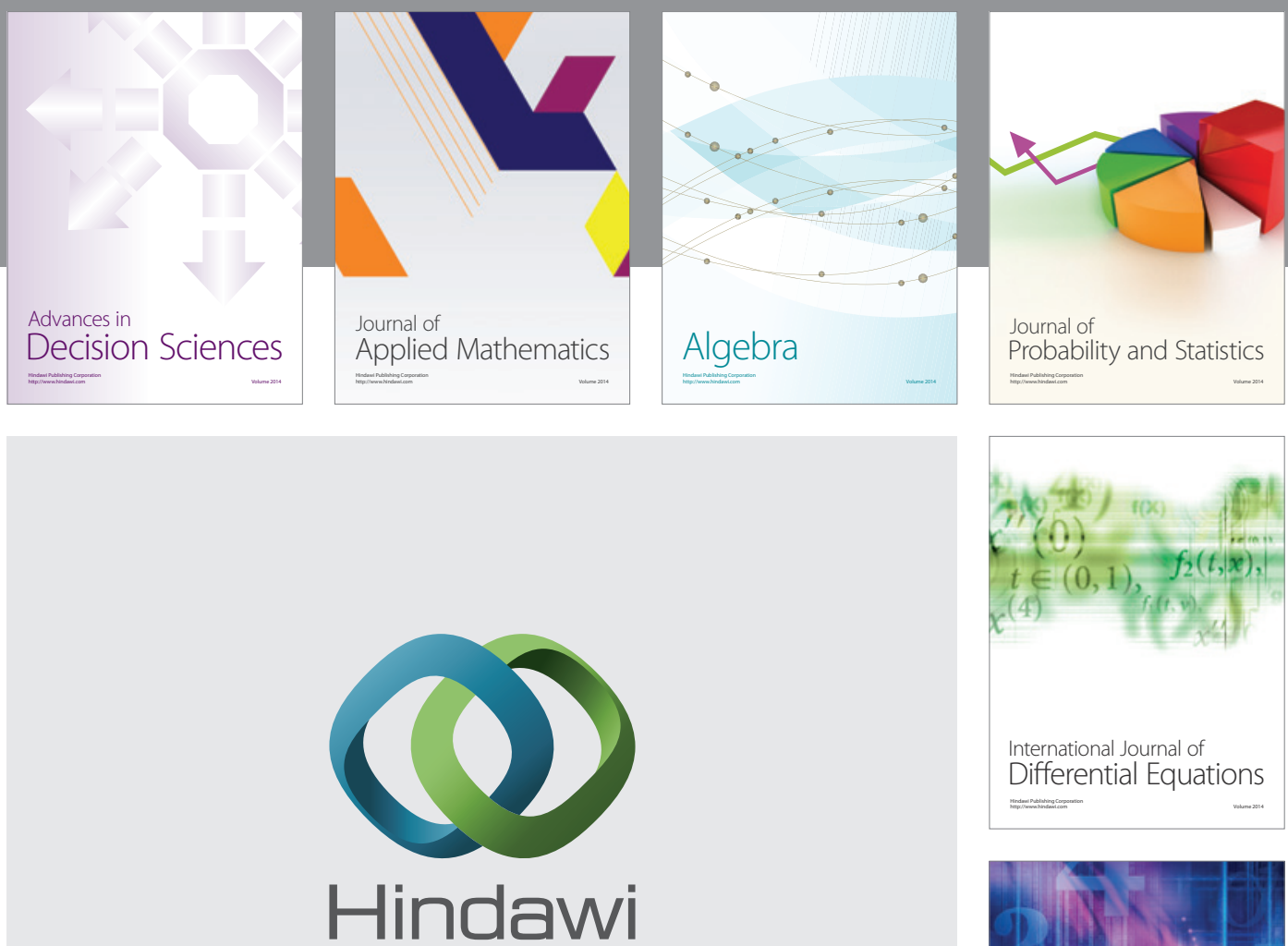

Submit your manuscripts at http://www.hindawi.com
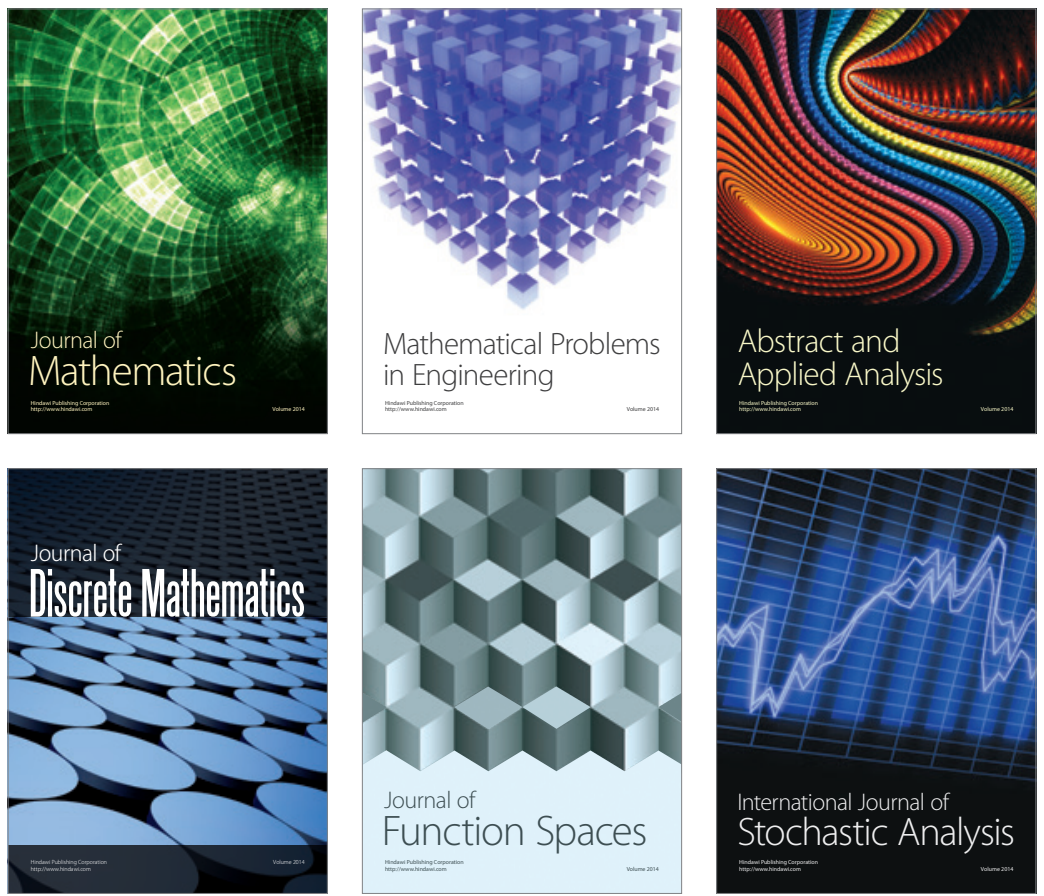

Journal of

Function Spaces

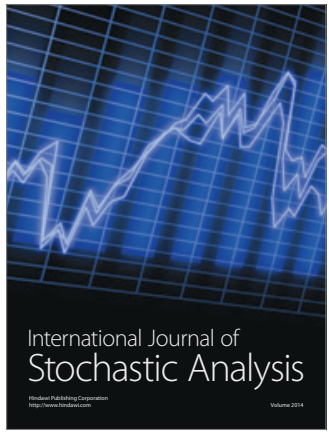

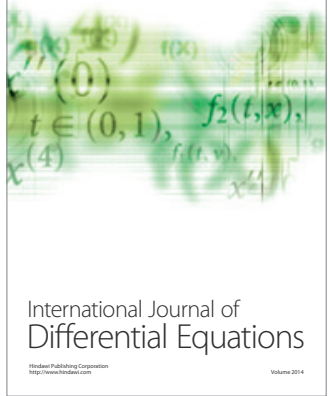
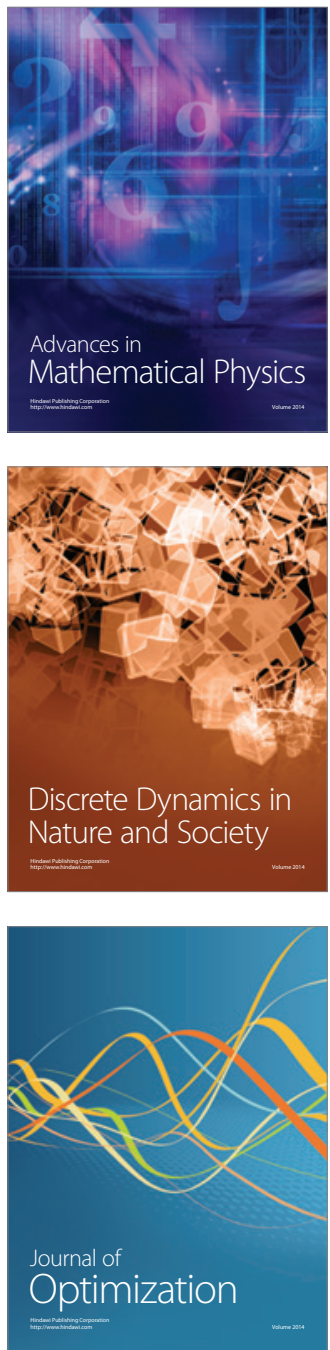